\title{
Towards understanding the lifespan extension by reduced insulin signaling: bioinformatics analysis of DAF-16/FOXO direct targets in Caenorhabditis elegans
}

\author{
Yan-Hui Li ${ }^{1}$ and Gai-Gai Zhang ${ }^{2}$ \\ ${ }^{1}$ Institute of Cardiovascular Sciences and Key Laboratory of Molecular Cardiovascular Sciences, Ministry of Education, Peking \\ University Health Science Center, Beijing, P. R. China \\ ${ }^{2}$ Special Medical Ward (Geratology Department), First Hospital of Tsinghua University, Beijing, P. R. China \\ Correspondence to: Yan-Hui Li, email: liyanhui@bjmu.edu.cn \\ Keywords: lifespan, C. elegans, insulin signaling, FOXO direct targets, bioinformatics, Gerotarget \\ Received: January 05, 2016 \\ Accepted: March 16, 2016 \\ Published: March 24, 2016
}

\section{ABSTRACT}

DAF-16, the $C$. elegans FOXO transcription factor, is an important determinant in aging and longevity. In this work, we manually curated FOXODB http://lyh.pkmu.cn/ foxodb/, a database of FOXO direct targets. It now covers 208 genes. Bioinformatics analysis on 109 DAF-16 direct targets in C. elegans found interesting results. (i) DAF16 and transcription factor PQM-1 co-regulate some targets. (ii) Seventeen targets directly regulate lifespan. (iii) Four targets are involved in lifespan extension induced by dietary restriction. And (iv) DAF-16 direct targets might play global roles in lifespan regulation.

\section{INTRODUCTION}

DAF-16, the C. elegans FOXO transcription factor, plays as a molecular switch in lifespan regulation [1]. When activated by reduced insulin signaling, it could extend C. elegans's lifespan by activating or inhibiting its downstream genes $[2,3]$. Presumably, these downstream genes largely determine how the lifespan can be extended. Yet, little is known about their positions in the regulatory network: which are directly regulated by DAF-16, and which are indirect targets.

To identify DAF-16 targets, various high throughput techniques have been used, such as microarray $[2,3]$, proteomics [4], and DamID (DNA adenine methyltransferase identification) [5]. Microarray and proteomics could identify DAF-16 downstream genes, but have difficult to figure out whether they are direct or indirect targets. DamID could identify DAF-16 direct targets in theory, but may have probability to identify false positives and negatives in practice [6].

Insulin signaling is remarkably conserved in $C$. elegans, Drosophila melanogaster and mammals, and reduced signaling of this pathway has been shown to extend lifespan in all of these animals [7]. For FOXO and its orthologs, there are different identifiers of genes, transcripts and proteins in different species. We called them all "FOXOs" hereafter. Currently, many experimentally validated FOXOs direct targets scattered in literatures $[6,8,9]$. Collecting these known targets, and then mapping them to $C$. elegans through orthologous analysis would be helpful for longevity research in $C$. elegans.

In this work, by manually reading literatures, we collected 208 experimentally validated FOXOs direct targets. Through orthologous mapping, we eventually got 109 DAF-16 direct targets in C. elegans. To make data easily accessible, we set up FOXODB (http://lyh.pkmu. $\mathrm{cn} /$ foxodb/). Bioinformatics analysis on the 109 targets revealed interesting results.

\section{RESULTS}

\section{FOXODB: a database of FOXO direct targets}

As shown in Figure 1, we searched PubMed with keywords "FOXOs" and found more than 2700 papers. We manually read the papers. When a gene was determined as a FOXOs direct target, key information was extracted and record in FOXODB (http://lyh.pkmu.cn/foxodb). 
Table 1: The seven DAF-16 direct targets that contain both DBE and DAE

\begin{tabular}{|l|l|l|}
\hline GeneID & DAF-16 binding element(DBE) & DAF-16 associate element(DAE) \\
\hline$d a f-2$ & [GTAAACA: 108$]$ & [CTTATCA: 85$]$ \\
\hline$m t l-1$ & [GTAAACA: 44$]$ & [CTTATCA: 417$]$ \\
\hline srp- 2 & [GTAAACA: 780$]$ & [CTTATCA: 165$]$ [TGATAAG: 448$]$ \\
\hline rars -1 & [TGTTAC: 925$]$ & [CTTATCA: 697$]$ \\
\hline T19C9.8 & [GTAAACA: 174$]$ & [CTTATCA: 54$]$ \\
\hline C25E 10.8 & [GTAAACA: 231$]$ & [CTTATCA: 935$]$ [TGATAAG: 267$]$ \\
\hline$d a f-16$ & [GTAAACA: 264$]$ & [TGATAAG: 46] \\
\hline
\end{tabular}

DAF-16 binding element (DBE): GTAAACA or TGTTTAC; DAF-16 associated element (DAE): TGATAAG or CTTATCA. [GTAAACA: 108] means that GTAAACA is located 108bp relative to transcription start site.

Rules for collecting a gene to FOXODB were strict. (i) The gene should be differentially expressed in FOXOs (+) versus FOXOs (-); (ii) FOXOs must be able to bind to the promoter of the gene; And (iii) only traditional experimental evidence(s) was adopted. Details can be found in Materials and Methods.

Currently, FOXODB covers 302 entries and 208 genes, including 35, 26, and 147 direct targets in C. elegans, Drosophila melanogaster and mammals, respectively. FOXODB is well designed and friendly to user (Figure 2). As in our previous works [10, 11], FOXODB was written in PHP (Hypertext Preprocessor). We believe FOXODB will be a valuable resource to the field.

\section{DAF-16 direct targets significantly overlaps with previous results}

Inparanoid is a database specially designed for orthologue analysis [12]. We used it to map FOXODB genes to C. elegans orthologs and got 109 DAF-16 direct targets eventually. (Supplementary Table 1 and 2).

The 109 targets significantly overlapped with genes found in previous works (Supplementary Table 3). For example, Murphy et al. found 514 differential expressed genes by comparing microarrays of daf-16 (+) versus daf-16 (-) [2], and 18 (2.8 in random chance, $p=0)$ of them overlapped with the 109 targets. Similar, Tepper et al. found 3,396 differential expressed genes [13], and 31 (18.5 in random chance, $p=0.0019$ ) of them overlapped with the 109 targets. Dong et al. found 86 proteins differentially expressed using proteomics [4], and $12(0.47$ under random, $p=0$ ) of them appeared in the 109 targets. Using DamID, 65 genes were found as potential DAF16 targets [5], and $6(0.31$ under random, $p=0)$ of them were in the 95 targets ( 14 genes were excluded from the 109 targets, since they were collected from this work. 95 =109-14). These results showed the 109 DAF-16 direct targets were reliable.

\section{DAF-16 and PQM-1 co-regulate some direct targets}

It has been reported that DAF-16 binding element (DBE), GTAAACA or TGTTTAC, and DAF-16associated element (DAE), TGATAAG or CTTATCA, enriched in DAF-16 regulated genes [2, 4, 13, 14]. The DBE was recognized by DAF-16, and the DAE by transcription factor PQM-1 [13]. Here, we searched both the DBE and the DAE in $1 \mathrm{~kb}$ promoter region (relative to TSS) of the 109 targets. As a result, 30 genes contained the DBE (Supplementary Table 4), 30 genes contained the DAE (Supplementary Table 5) and 7 genes contained both (Table 1). Recent work showed that DAF-16 could even recognize DAE [15]. Taken together, these results indicated DAF-16 and PQM-1 at least co-regulate some direct targets.

\section{Seventeen DAF-16 direct targets directly regulated lifespan}

GenAge [16], an useful longevity research resource, covers 681 longevity genes in C. elegans. Compared with the 109 targets, 17 genes overlapped, significantly higher than 3.71 under random, $p=0$ (Table 2). Of the 17 genes, 10 were obtained by orthologous mapping. This means they were for the first time known as DAF-16 direct targets that regulate lifespan.

According to GenAge, longevity genes were classified into anti-longevity and pro-longevity [16, 17]. Knockout or suppression of anti-longevity gene, or overexpression of pro-longevity gene resulted in lifespan extension, whereas the opposite interventions led to reduction of lifespan [16, 17]. Of the 17 genes, 10 were classified as anti-longevity, 6 as pro-longevity, and one, sod-2, was not classified. We observed their expressions in Murphy et al.'s microarrays as mentioned above [2]. gpd-2, pck-2 and nnt-1 were up-regulated, and $d o d-17$ was down-regulated in daf-16(+) vs. daf-16(-). Since gpd2 and $p c k-2$ were anti-longevity, their up-regulations were not expected in $d a f-16(+)$ animals. Thus, we inferred that 


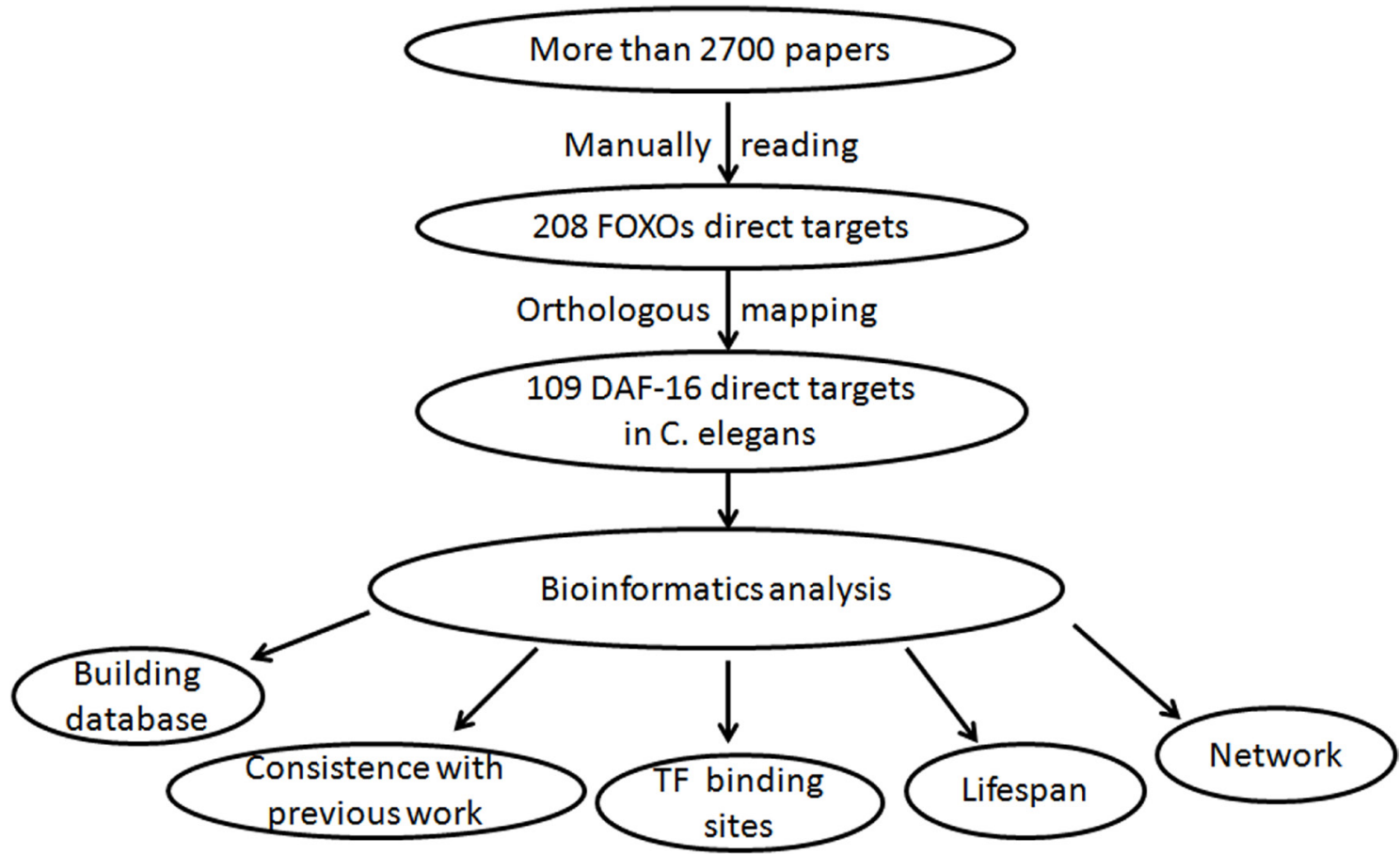

Figure 1: Workflow. We first searched PubMed with FOXOs keywords and found more than 2700 papers. Through manually reading these papers, we got 208 FOXOs direct targets. Inparanoid was used to map FOXOs targets to their orthologs in C. elegans. And 109 genes were found eventually. Bioinformatics analysis on the 109 genes were done including comparison with previous results, transcription factor binding site enrichment, lifespan regulation and network topological feature analysis. And we built a database to make all data easily accessible.

\section{FOXODB: a Database of FOXOs Direct Targets}

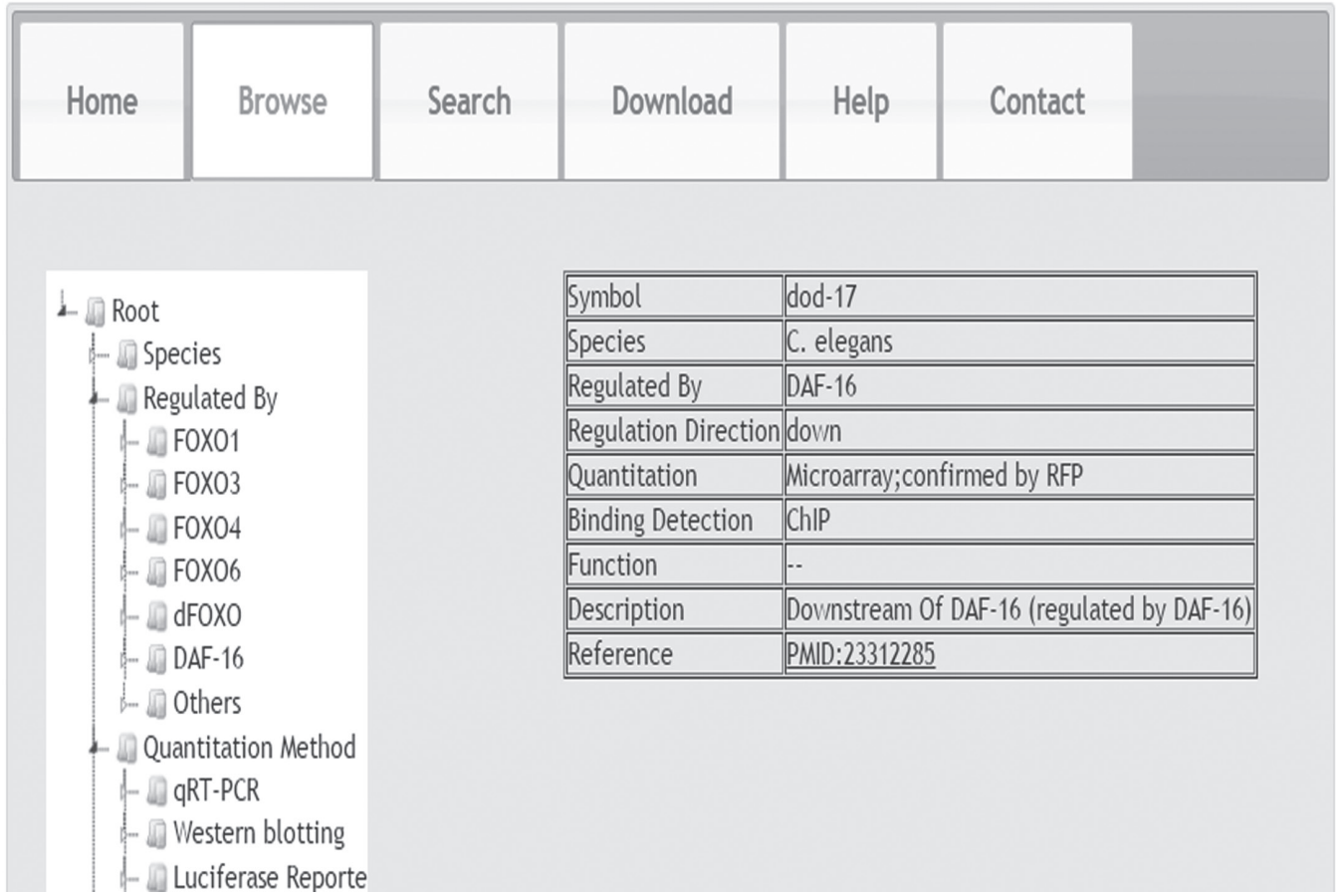

Figure 2: FOXODB database. FOXODB is a database aiming at collecting experimentally verified direct targets of FOXOs. FOXODB is well designed and friendly to user. User could easily browse, search and download the genes. 
Table 2: The 17 DAF-16 direct targets that directly regulate lifespan

\begin{tabular}{|l|l|l|}
\hline Symbol & Longevity influence & Orthologous mapping \\
\hline din-1 & Pro-Longevity & Yes \\
\hline $\operatorname{lgg}-1$ & Pro-Longevity & Yes \\
\hline$m d h-1$ & Pro-Longevity & No \\
\hline$n n t-1 *$ & Pro-Longevity & No \\
\hline$p r d x-3$ & Pro-Longevity & Yes \\
\hline$d a f-16$ & Pro-Longevity & Yes \\
\hline$a c o-2$ & Anti-Longevity & Yes \\
\hline$a g e-1$ & Anti-Longevity & Yes \\
\hline$d a f-2$ & Anti-Longevity & Yes \\
\hline$d a f-7$ & Anti-Longevity & Yes \\
\hline$d o d-17 \#$ & Anti-Longevity & No \\
\hline gpd-2* & Anti-Longevity & No \\
\hline$u b h-4$ & Anti-Longevity & No \\
\hline$p c k-2 *$ & Anti-Longevity & No \\
\hline W09D10.3 & Anti-Longevity & Yes \\
\hline lars-2 & Anti-Longevity & Yes \\
\hline sod-2 & Unclear & No \\
\hline
\end{tabular}

* and \# represent up and down regulated in $d a f-16(+)$ versus $d a f-16(-)$, respectively. Longevity genes were classified into anti-longevity and pro-longevity. Knockout or suppression of anti-longevity gene, or overexpression of pro-longevity gene resulted in lifespan extension, whereas the opposite interventions led to reduction of lifespan. Orthologous mapping describes whether the gene was gotten from orthologous mapping (Yes) or not (NO).

knocking out or down either gpd-2 or pck-2 in daf-16(+) might further extend lifespan. Fortunately, this inference has been validated in previous work [4]

\section{Four DAF-16 direct targets were involved in lifespan extension induced by dietary restriction}

Many dietary restriction methods could extend $C$. elegans's lifespan [18]. Some of them such as eat mutation or some forms of bacterial dilution do not require DAF-16, while some other forms of bacterial dilution and peptone dilution require DAF-16 [18]. Thus, it was interesting to know whether DAF-16 direct targets were involved in lifespan extension induced by dietary restriction. GenDR, a database collecting lifespan-regulating genes related to dietary restriction, covers 48 genes in C. elegans [19]. Here, we compared them with the 109 targets and found 4 overlapping genes: age-1, hsp-12.6, daf-16 and daf-2. This was significantly higher than 0.26 under random, with $p=$ 1.35E-4. This result supported that some dietary restriction methods required DAF-16 for lifespan extension.

\section{DAF-16 direct targets might play global roles in lifespan regulation}

Proteins do not function in isolation but through interaction with each other. And from network view, the more interaction partners (higher degrees) one protein has, the more important the protein might be. Here, we studied the degrees of the 109 targets, and found that the average degree is 17.77 , significantly higher than 11.85 , the average degree for other proteins in the network ( $p=$ 0.0014, Kolmogorov-Smirnov test, KS test for short). As analyzed above, 17 targets directly regulated lifespan. The average degree for them is the highest, 36.31 (see Figure $3 \mathrm{~A}$ ). This result was consistent with our previous work, the degrees of longevity genes tend to be higher than that of non- longevity genes [20].

$K$-core, another network index, takes into account not only the number of direct neighbors but also the placement of a protein in the network. It assumed that centrally located proteins are more important than the peripheral ones [21]. As shown in Figure 3B, the 109 targets have an average $K$-core 7.59 , significantly higher than 7.37, the average for other proteins in the network ( $p$ $=8.4 * \mathrm{E}-4, \mathrm{KS}$ test). And the 17 lifespan-regulating targets had the highest average $K$-core 10 .

To know whether DAF-16 direct targets function through cooperation with each other, we computed for each protein the 'target neighbor ratio'. It is the ratio of the number of interaction partners that belong to the DAF16 direct targets to its degree [22]. As shown in Figure 3C, DAF-16 direct targets tend to directly interact with each other $(p=2.7 * \mathrm{E}-4, \mathrm{KS}$ test $)$.

In all, these results revealed that DAF-16 direct targets tended to have more interaction neighbors, locate 


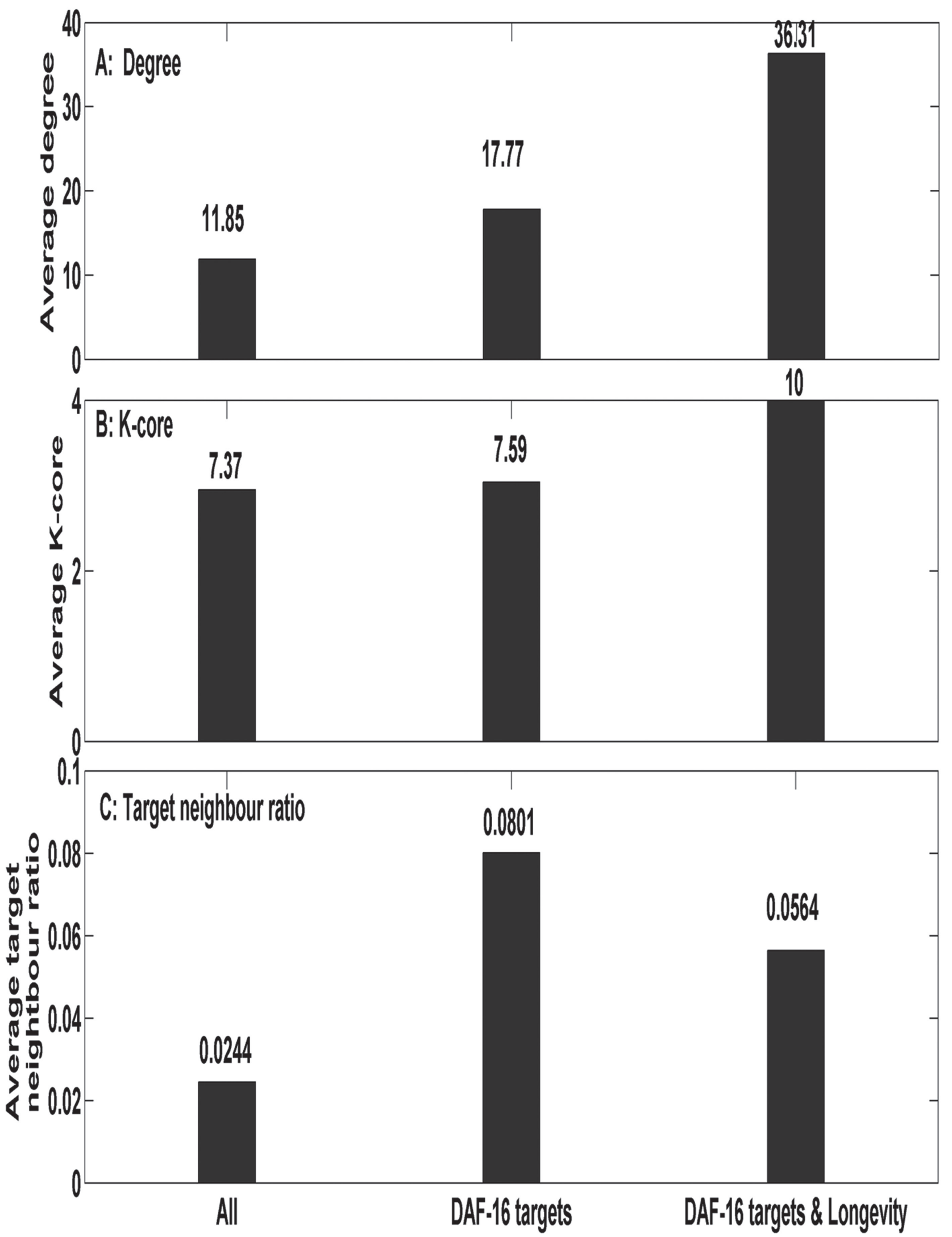

Figure 3: Network topological features of DAF-16 direct targets. The network topological features of DAF-16 direct targets were shown. 'All' represents all proteins in the network. 'DAF-16 targets' represents the 109 DAF-16 direct targets in C. elegans. 'DAF16 targets \& longevity' represents the DAF-16 direct targets that regulate lifespan. Figure A is the average degree, Figure B is the average $K$-core, and Figure $\mathbf{C}$ is the average of target neighbor ratio. 
network center and interact with each other. This implied that DAF-16 direct targets might play global roles in lifespan regulation.

\section{DISCUSSION}

In this work, we manually curated FOXODB by reading literatures. It now covers 208 FOXOs direct targets. To our knowledge, this is the largest. 109 DAF16 direct targets in C. elegans were found by orthologous mapping. And 17 of them directly regulated lifespan. These are also important data to the field.

We searched DAF-16 binding element (DBE) in $1 \mathrm{~kb}$ promoter region of the 109 DAF-16 direct targets, and found 30 of them contained the DBE (GTAAACA or TGTTTAC) while the others not. It was difficult to understand why so many DAF-16 direct targets did not contain DBE. For explanation, first, different works used different DBE motifs [2-4]. It was hard to know which one was correct. We chose a strict DBE motif and thus resulted in few sequence matches. If using a loose DBE motif, more genes with DBE could be found. For example, when using DBE, RTAAAYA, $\mathrm{R}=\mathrm{A} / \mathrm{G}, \mathrm{Y}=\mathrm{C} / \mathrm{T}$, as in previous work [3], 91 of the 109 targets would contain the DBE in $1 \mathrm{~kb}$ promoter region. Second, we only searched the $1 \mathrm{~kb}$ promoter region. Some DBE may locate outside of the region and thus not be found.

We did the first network analysis on DAF-16 direct targets. The results showed they tended to be higher in degree, locate network center and directly interact with each other. The protein interactions used for network analysis include several kinds of interactions such as physical interaction, genetic interaction and predicted interaction. However, it's worth noting that some of the interactions might be collected from literatures. Thus, the more a gene being studied, the more likely the gene has higher degree. Though the collected interactions may be only a small part of the whole data, we still cannot exclude the possibility that this might affect the results.

\section{MATERIALS AND METHODS}

\section{Data source}

The gene sequences were downloaded from WormBase, version 220. Protein interaction network was obtained from our previous work [20]. The network was constructed by integrating different kinds of interactions including physical interactions, genetics interactions and predicted interactions, covering a total of 7,219 proteins and 41, 132 edges [20].

\section{Workflow}

As shown in Figure 1, we searched PubMed with 'FOXOs' and found more than 2700 papers. We manually read the papers and found 208 FOXOs direct targets. Inparanoid is a database specially designed for orthologous analysis [12]. We used it to map the 208 targets to their orthologs in $C$. elegans and finally got 109 genes. Bioinformatics analysis on this list were done including comparison with previous results, transcription factor binding site enrichment, lifespan regulation and network topological feature analysis. We built a database to make all data easily accessible.

\section{Database creation}

To collect FOXOs direct targets, we searched PubMed using keywords: 'FOXO1, FOXO3, FOXO4, FOXO6, AFX, FKHR, dFOXO and DAF-16'. Then we manually read the papers and retrieved gene symbol, species, quantitation methods, DNA binding detection methods and function descriptions et al.. Strict rules were used to determine whether a gene was a FOXOs direct target or not. (i) The gene should be differentially expressed in FOXOs (+) VS. FOXOs (-). The quantitation method should be traditional such as RT-PCR or western blotting. And if high throughput quantitation method such as microarray was used, the result must be further confirmed by other technique like GFP (Green Fluorescent Protein). (ii) There must be evidence showing that FOXOs could bind to the promoter region of the gene, like ChIP (chromatin immunoprecipitation) and EMSA (Electrophoretic mobility shift assay), or mutating the FOXOs binding site could significantly change the gene expression.

\section{Hypergeometric model}

The hypergeometric model was used for calculating the significance of two gene sets with a certain number of overlapping genes. The P-value is calculated as follows:

$$
P \text { value }=1-\sum_{i=0}^{k-1} \frac{\left(\begin{array}{c}
m \\
i
\end{array}\right)+\left(\begin{array}{c}
N-m \\
n-i
\end{array}\right)}{\left(\begin{array}{l}
N \\
n
\end{array}\right)}
$$

$N$ : Number of genes in C. elegans genome, 20,000 was used for approximation in this work.

$m$ : Number of genes in gene set 1 .

$n$ : Number of genes in gene set 2 .

$k$ : Number of overlapping genes between the $m$ genes and the $n$ genes. 
Table 3: Formal representation of graph measures

\begin{tabular}{|l|l|l|}
\hline Name & Function & Descriptions \\
\hline Degree & $K_{i}$ & the number of interaction partners of node i \\
\hline $\begin{array}{l}\text { Target neighbor } \\
\text { ratio }\end{array}$ & $K^{p}{ }_{i}$ & $\begin{array}{l}K^{p}{ }_{i} \text { is the number of links between node i and proteins encoded } \\
\text { by DAF-16 direct targets }\end{array}$ \\
\hline$K$-core & $K$ & $\begin{array}{l}\text { A } K \text {-core of a graph can be obtained by recursively removing all } \\
\text { nodes with a degree less than } K, \text { until all nodes in the remaining } \\
\text { graph have a degree at least } K .\end{array}$ \\
\hline
\end{tabular}

Functions are the definitions of the topological features. Descriptions give explanations for symbols in the definitions.

\section{Kolmogorov-Smirnov test}

In statistics, the two-sample Kolmogorov-Smirnov test is one of the most useful and general nonparametric methods for comparing two samples, as it is sensitive to differences in both location and shape of the empirical cumulative distribution functions of the two samples. In this work, two-sample $K S$ test was used to compare the network topological features of DAF-16 direct targets and that of the remaining genes in C. elegans.

\section{Network topological features}

The network topological features degree and $K$-core were computed by using an R package igraph [23]. The definitions for them can be found in Table 3 .

\section{GRANT SUPPORT}

This work was supported by the National Natural Science Foundation of China (grant nos. 81300253).

\section{Authors' contributions}

Yan-Hui Li conceived and designed the experiments. Yan-Hui Li performed the experiments and wrote the paper. Gai-Gai Zhang collected part of the FOXOs target genes. Gai-Gai Zhang revised the paper. Both authors read and approved the final manuscript.

\section{CONFLICTS OF INTEREST} interests.

All the authors declare that they have no competing

\section{REFERENCES}

1. Kenyon C, Chang J, Gensch E, Rudner A and Tabtiang R. A C. elegans mutant that lives twice as long as wild type. Nature. 1993; 366:461-464.
2. Murphy CT, McCarroll SA, Bargmann CI, Fraser A, Kamath RS, Ahringer J, Li H and Kenyon C. Genes that act downstream of DAF-16 to influence the lifespan of Caenorhabditis elegans. Nature. 2003; 424:277-283.

3. McElwee J, Bubb K and Thomas JH. Transcriptional outputs of the Caenorhabditis elegans forkhead protein DAF-16. Aging Cell. 2003; 2:111-121.

4. Dong MQ, Venable JD, Au N, Xu T, Park SK, Cociorva D, Johnson JR, Dillin A and Yates JR, 3rd. Quantitative mass spectrometry identifies insulin signaling targets in $\mathrm{C}$. elegans. Science. 2007; 317:660-663.

5. Schuster E, McElwee JJ, Tullet JM, Doonan R, Matthijssens F, Reece-Hoyes JS, Hope IA, Vanfleteren JR, Thornton JM and Gems D. DamID in C. elegans reveals longevityassociated targets of DAF-16/FoxO. Mol Syst Biol. 2010; 6:399.

6. Murphy CT. The search for DAF-16/FOXO transcriptional targets: approaches and discoveries. Exp Gerontol. 2006; 41:910-921.

7. Kenyon C. The plasticity of aging: insights from long-lived mutants. Cell. 2005; 120:449-460.

8. van der Vos KE and Coffer PJ. The extending network of FOXO transcriptional target genes. Antioxid Redox Signal. 2011; 14:579-592.

9. Puig $\mathrm{O}$ and Mattila J. Understanding Forkhead box class $\mathrm{O}$ function: lessons from Drosophila melanogaster. Antioxid Redox Signal. 2011; 14:635-647.

10. Li Y, Wang C, Miao Z, Bi X, Wu D, Jin N, Wang L, Wu H, Qian K, Li C, Zhang T, Zhang C, Yi Y, Lai H, Hu Y, Cheng L, et al. ViRBase: a resource for virus-host ncRNAassociated interactions. Nucleic Acids Res. 2015; 43:D578582 .

11. Li YH, Zhang G and Cui Q. PPUS: a web server to predict PUS-specific pseudouridine sites. Bioinformatics. 2015; 31:3362-3364

12. Ostlund G, Schmitt T, Forslund K, Kostler T, Messina DN, Roopra S, Frings O and Sonnhammer EL. InParanoid 7: new algorithms and tools for eukaryotic orthology analysis. Nucleic Acids Res. 38:D196-203.

13. Tepper RG, Ashraf J, Kaletsky R, Kleemann G, Murphy CT and Bussemaker HJ. PQM-1 complements DAF-16 as a key 
transcriptional regulator of DAF-2-mediated development and longevity. Cell. 2013; 154:676-690.

14. Budovskaya YV, Wu K, Southworth LK, Jiang M, Tedesco P, Johnson TE and Kim SK. An elt-3/elt-5/elt-6 GATA transcription circuit guides aging in C. elegans. Cell. 2008; 134:291-303.

15. Zhang P, Judy M, Lee SJ and Kenyon C. Direct and indirect gene regulation by a life-extending FOXO protein in $\mathrm{C}$. elegans: roles for GATA factors and lipid gene regulators. Cell Metab. 2013; 17:85-100.

16. Tacutu R, Craig T, Budovsky A, Wuttke D, Lehmann G, Taranukha D, Costa J, Fraifeld VE and de Magalhaes JP. Human Ageing Genomic Resources: integrated databases and tools for the biology and genetics of ageing. Nucleic Acids Res. 2013; 41:D1027-1033.

17. Budovsky A, Tacutu R, Yanai H, Abramovich A, Wolfson $\mathrm{M}$ and Fraifeld V. Common gene signature of cancer and longevity. Mech Ageing Dev. 2009; 130:33-39.

18. Greer EL and Brunet A. Different dietary restriction regimens extend lifespan by both independent and overlapping genetic pathways in C. elegans. Aging Cell. 2009; 8:113-127.
19. Wuttke D, Connor R, Vora C, Craig T, Li Y, Wood S, Vasieva O, Shmookler Reis R, Tang F and de Magalhaes JP. Dissecting the gene network of dietary restriction to identify evolutionarily conserved pathways and new functional genes. PLoS Genet. 2012; 8:e1002834.

20. Li YH, Dong MQ and Guo Z. Systematic analysis and prediction of longevity genes in Caenorhabditis elegans. Mech Ageing Dev. 2010; 131:700-709.

21. Wuchty S and Almaas E. Peeling the yeast protein network. Proteomics. 2005; 5:444-449.

22. $\mathrm{Xu} \mathrm{J}$ and Li Y. Discovering disease-genes by topological features in human protein-protein interaction network. Bioinformatics. 2006; 22:2800-2805.

23. Csardi G NT. The igraph software package for complex network research. InterJournal, Complex Systems. 2006; http://igraph.sf.net. 